\title{
Fundamental and essential techniques for supermicrosurgical lymphaticovenular anastomosis: the art of Isao Koshima's supermicrosurgery
}

\author{
Yukio Seki, Akiyoshi Kajikawa \\ Department of Plastic and Reconstructive Surgery, St. Marianna University School of Medicine, Kawasaki, Kanagawa 216-8511, \\ Japan.
}

Correspondence to: Dr. Yukio Seki, Department of Plastic and Reconstructive Surgery, St. Marianna University School of Medicine, 2-16-1 Sugao, Miyamae-ku, Kawasaki, Kanagawa 216-8511, Japan. E-mail: yukioseki.pla@gmail.com

How to cite this article: Seki Y, Kajikawa A. Fundamental and essential techniques for supermicrosurgical lymphaticovenular anastomosis: the art of Isao Koshima's supermicrosurgery. Plast Aesthet Res 2021;8:44. https://dx.doi.org/10.20517/23479264.2021.74

Received: 21 Jun 2021 First Decision: 26 Jul 2021 Revised: 4 Aug 2021 Accepted: 24 Aug 2021 First online: 26 Aug 2021

Academic Editors: Matthew L lorio, Isao Koshima Copy Editor: Yue-Yue Zhang Production Editor: Yue-Yue Zhang

\begin{abstract}
Lymphaticovenular anastomosis (LVA) is a highly effective, minimally invasive surgical treatment for lymphedema. The effect of LVA appears immediately after the creation of lymph-to-venous pathway. However, the long-term outcome of LVA is not always promising when the lymph-to-venous anastomosis has any potential risk of occlusion. The reasons of postoperative LVA occlusions are considered both a technical matter in performing LVA and a strategic matter in preoperative planning. This report focuses on intraoperative techniques of LVA to avoid postoperative occlusions. Depending on the types of undesirable surgical procedures, lymphaticovenular anastomoses are at risk of future occlusions in early, mid-, or late-postoperative course. The authors describe fundamental and essential techniques to perform supermicrosurgical LVA, and the true concept of Isao Koshima's supermicrosurgery, in which the pith and marrow of the doctrine is not only the way of handling the small vessels or needles, but also the surgeons' skills to feel intima of the vessels and lymphatic flow itself.
\end{abstract}

Keywords: Lymphedema, lymphaticovenular anastomosis, supermicrosurgery, one hand suture technique, surgery 


\section{INTRODUCTION}

Lymphedema is a chronic and progressive disease which causes physical and psychological morbidity in upper and lower extremities ${ }^{[1-4]}$. Lymphaticovenular anastomosis (LVA) is a highly effective, minimally invasive surgical treatment for lymphedema ${ }^{[5-14]}$, in which a new lymphatic pathway is created by anastomosing the peripheral lymphatic vessel directly into the subcutaneous vein. The surgical effect of LVA appears immediately after the surgery, because of physiological changes of lymph dynamics in the affected limbs ${ }^{[9-12]}$.

The clinical effect of LVA usually continues for a longtime. However, LVA is under the risk of future occlusions $^{[1,5,16]}$. That is why the long-term outcome of LVA is not always guaranteed. There are many potential risks of postoperative LVA occlusion, which include lymphatic vessels' degenerations, lymphedema severity, postoperative managements, surgical site infection, patients' compliance, selection of LVA location, changes of postoperative lymphatic dynamics, and surgical procedures themselves ${ }^{[8-18]}$. Among these reasons for LVA occlusions, selection of LVA locations and fine procedures in LVA are particularly important for microsurgeons to achieve good clinical results ${ }^{[7-14]}$.

Because surgical skills of LVA require one of the finest techniques in microsurgery, only minor mistakes could result in LVA failure. Depending on the type of unsuitable LVA procedures, there is a risk of LVA occlusion in early, mid-, or late-postoperative course. Inaccuracy of the anastomosis and structural disturbance between the lymphatic vessel and the subcutaneous vein are potential risks for early- or midpostoperative occlusion of LVA. In contrast, instability of decreased lymphatic flow at the anastomosis, which is induced by improvement of lymphedema, is considered to cause mid- or late-postoperative LVA occlusion. Although there are many reasons for LVA occlusions, technically indued LVA occlusions are considered to be avoidable with the development of fine procedures.

In this paper, the fundamental and essential techniques to perform supermicrosurgical LVA are described to improve accuracy of intraoperative procedures with the basic theory of Isao Koshima's supermicrosurgical LVA. In addition, the senior author's current techniques in LVA procedures which have been explored to improve the therapeutic effect of LVA based on Isao Koshima's teachings are described with video files (see the Supplementary Materials).

\section{FUNDAMENTAL TECHNIQUES IN SUPERMICROSURGERY}

The concept of supermicrosurgery has been advocated by Isao Koshima since the 1990s to anastomose small vessels less than $0.80 \mathrm{~mm}$ in diameter using operating microscopes ${ }^{[1,200]}$. Because recent advancements in operating microscopes and surgical instruments make anastomosis of small vessels relatively easy, the true supermicrosurgical techniques are mostly applied to anastomose small vessels less than $0.50 \mathrm{~mm}$ in diameter for lymphatic surgery, fingertip replantation, nerve flap, and perforator-to-perforator tissue transplantation nowadays ${ }^{[8-14,20]}$.

Supermicrosurgery is sometimes misunderstood just as special techniques to handle small vessels with operating microscopes at high magnification. However, the true concept of supermicrosurgery is based on skills to feel the intima of vessels or lymphatic flow itself by microsurgeons' finger sensation via the tip of the needle to perform intima-to-intima anastomosis between very small vessels.

These finger sensation skills make it possible to anastomose small vessels using a normal operating microscope with normal magnification function: the senior author usually selects 12-18 times magnification of operating microscope to anastomose vessels over $0.25 \mathrm{~mm}$ in diameter ${ }^{[10-12]}$. When this finger sensation 
skill of feeling is closely linked with the visual information which is obtained from the operating microscope, supermicrosurgeons can finally anastomose very small lymphatic vessels of around $0.10 \mathrm{~mm}$ in diameter. Needle selection is important too. Our selection of needle is 11-0 nylon suture for vessels of around $0.30-0.80 \mathrm{~mm}$ in diameter, $12-0$ nylon suture for 0.20-0.45 $\mathrm{mm}$ in diameter, and 12-0 s nylon suture for less than $0.20 \mathrm{~mm}$ in diameter according to the hardness of the vessels.

The way of Isao Koshima's supermicrosurgical anastomosis is called the "one-hand suture technique", because supermicrosurgeons create anastomosis as if they only used the right hand to make each suture. However, this description of the supermicrosurgery is not correct in reality. In supermicrosurgery, surgeons utilize both their hands equally to feel the lumen of the small vessels. The left hand has essential work.

\section{ONE-HAND SUTURE TECHNIQUE}

The most important technique in supermicrosurgery is the one-hand suture technique. The name of this technique is from the extraordinal suture style as if microsurgeons only used their right hand in anastomosing procedure for handling the needle. However, the left hand is also important for successful LVA. This technique is the way to feel the intima of small vessels and movement of blood and lymphatic fluid in the anastomosing vessel using surgeons' five senses.

Understanding the technical difference between supermicrosurgery and traditional microsurgery is important to know the spirit of Isao Koshima's supermicrosurgery. In traditional microsurgery, the basic microsurgical technique is an effective left-hand work to anastomose the relatively large vessels safely. The left hand is used to insert the tips of forceps into the anastomosing vessel to dilate and keep the lumen in non-supermicrosurgery [Figure 1]. The intima-to-intima coaptation manner in the anastomosis can be performed by the insertion of the left-hand forceps into the anastomosing vessel on each suture without picking the back wall up.

In contrast, supermicrosurgical anastomoses using small vessels less than $0.50 \mathrm{~mm}$ in diameter require different usage of the surgeon's hands [Figure 2]. In small vessels, only one side tip of the forceps can be inserted and dilate the lumen. The traditional safe microsurgical technique using both tips of the forceps is not applicable in anastomosing small vessels. In supermicrosurgery, the surgeons' skills to feel the intima of vessels and fluid flow itself are utilized as a safe way to create fine anastomosis.

To feel vessels and flow, the left-hand forceps are utilized to hold the last suture or the adventitia of lymphatic vessel and vein during making sutures. This left hand is working both as a traction maker and as a sensor of the special feeling by controlling the shape of the vessels and fluid movement in the anastomosing vessels with proper tensioned traction. Whether the microsurgeon can feel the lumen and flow in the vessels totally relies on the strength and direction of the left-hand traction and the right-hand sensation at the tip of the needle via the needle holder. Supermicrosurgeons can complete the anastomosis using the finger sensation of both their hands without inserting the tips of forceps into the anastomosing vessels.

\section{SUPERMICROSURGICAL LVA}

LVA is a challenging procedure even for expert microsurgeons. One of the main technical difficulties of LVA is the size of small lymphatic vessels. The lymphatic vessel adopted in LVA is small (mainly 0.20-0.70 $\mathrm{mm}$ in diameter), which is difficult detect, to dissect, and anastomose even in an operating microscopic magnification. 


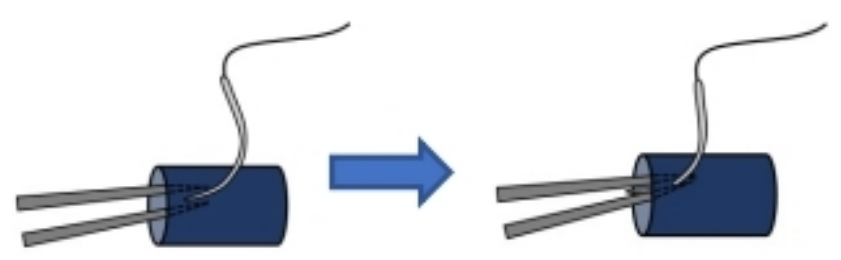

Figure 1. Safe procedure in traditional (non-supermicrosurgery) microsurgery. In traditional microsurgery (non-supermicrosurgery), the left hand is used to insert the tips of forceps into the anastomosing vessel to dilate and keep the lumen. Intima-to-intima anastomosis can be performed in each suture by inserting the left-hand forceps into the lumen to not pick up the back wall.

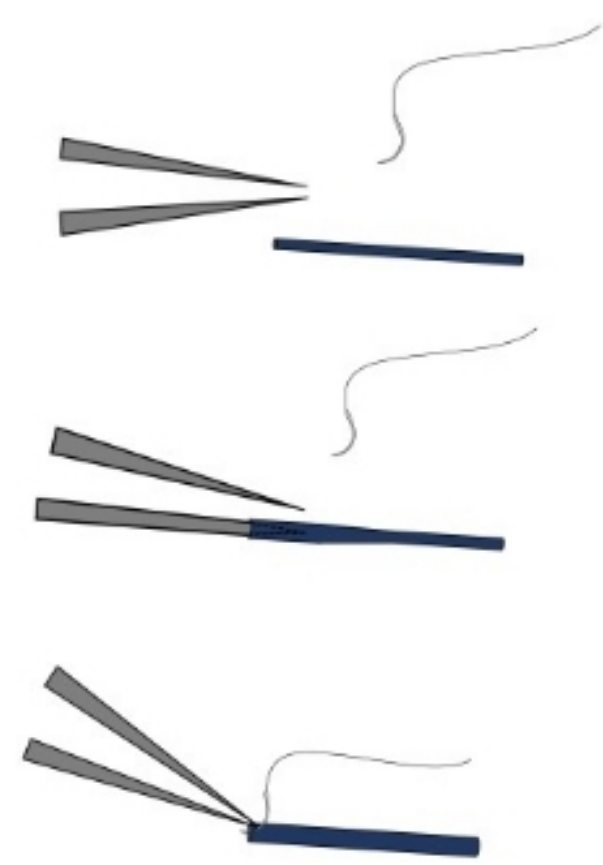

Figure 2. The left-hand forceps in supermicrosurgery to perform intima-to-intima anastomosis. In handling small vessels less than $0.5 \mathrm{~mm}$ in diameter, only one side tip of the forceps can be inserted and dilate the lumen. In supermicrosurgery, the surgeon's left hand always holds the last suture or the adventitia of the lymphatic vessel and vein when making suture to feel the intima of vessels and lymphatic flow itself. In addition, the right hand plays an important role to feel the lumen of the anastomosing vessel, cooperating with the left hand's feeling by sensing the tip of the needle via the needle holder.

Furthermore, severe lymphedema patients reveal degeneration of lymphatic vessels ${ }^{[8,17,18]}$. Degenerated lymphatic vessels are fragile and easily rupture even under fine procedures of microsurgical dissection. More delicate procedures are required in severe lymphedema because the lumen of degenerated lymphatic vessels become very narrow, and the sclerosed endometrium is easily peeled off. In this situation, skills to feel the flow of lymph and the adequate tensioned left-hand traction are necessary to perform anastomosis while searching for the true lumen from the easily peeled intima of the sclerosed lymphatic vessel [Figure 3].

After the LVA creation, it is necessary to remove any factors which can block or disturb the flow of lymph in LVA before closing the wound.

\section{Techniques in LVA STEP 1: incision and fixation of the surgical site}

Based on Isao Koshima's supermicrosurgical theory, the senior author's current step-by-step LVA procedures are described as follows. 

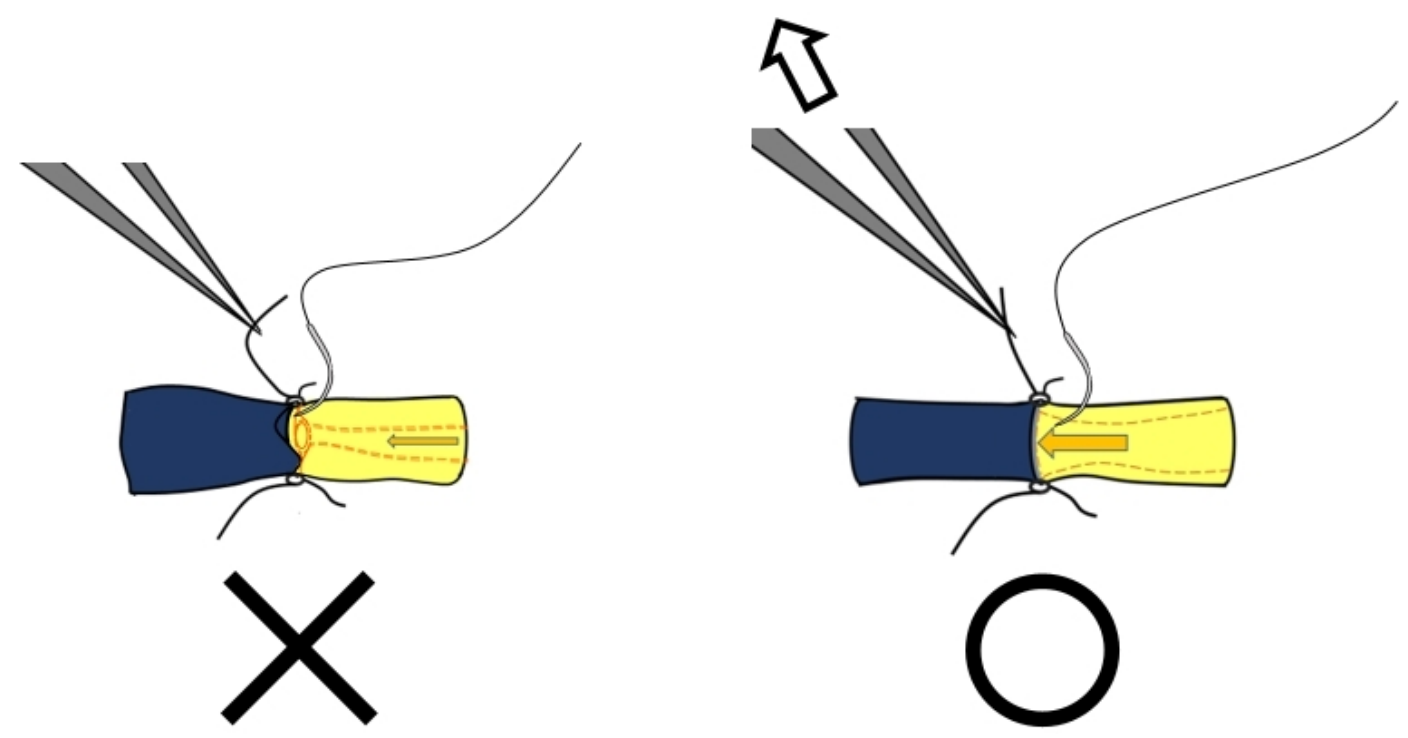

Figure 3. Supermicrosurgical one-hand suture technique for severe lymphedema. In the one-hand suture technique, the left hand is working both as a traction maker and a sensor of the special senses. An adequate tension of the left-hand traction is the only way to control the shape of the vessels and fluid movement in the anastomosing vessel to feel intima and fluid movement in the vessel by the microsurgeon's finger sensations (right). The range of adequate direction and tension of the left-hand traction is very narrow for performing one-hand suture technique. Intima-to-intima anastomosis of a small vessel is difficult if the surgeon's left hand cannot create proper retraction (left).

In peripheral lymphedema, the incision site for LVA could be selected from all areas of the limbs when lymphatic vessels are running in the incision site closely with the subcutaneous veins. However, location selection of LVA incision directly affects the therapeutic effect ${ }^{[8-14]}$. Therefore, it is important to update the treatment strategy based on previously reported LVA theories and imaging study including ICG lymphangiography and ultrasonography ${ }^{[7-14,21-24]}$.

Anatomically, lymphatic vessels have collaterals developed in the extremities, so there is no risk of new lymphedema occurring by cutting the lymphatic vessels at the small LVA incision. Too long incision at the limbs should be avoided for LVA because cutting the collaterals of all superficial lymphatic vessels can reduce the total flow of lymph at the lymphedematous extremities: all our LVA incisions have been within $3.0 \mathrm{~cm}$ in length (mostly $1.0-2.5 \mathrm{~cm}$ in length) thus far, and no patients have revealed abnormal lymph dynamics by the LVA incisions.

To achieve successful LVA, fixation of the surgical site is essential. The tightly fixed incision not only stabilizes the vessels to be dissected, but also allows stable movement of the surgeon's hand, thus suppressing the surgeon's fingers from slight tremor. Eliminating hand tremor of the surgeon is not a prerequisite for performing LVA. Beautiful anastomoses are created by many expert supermicrosurgeons even with slight tremor in microscopic view. This means that the special skill of finger sensations is more important than eliminating slight tremor. However, anastomosing lymphatic vessels less than $0.15 \mathrm{~mm}$ in diameter require the microsurgeons' hand to be without tremor, and the procedures is impossible without tight fixation of the incision.

Because the incision of LVA is small $(1.0-2.5 \mathrm{~cm})$, needle hook-type retractors are the most suitable for LVA [Figure 4]. The senior author usually uses four hook-type retractors at each incision. Under the fixation, LVA can be performed easily even with local anesthesia. 

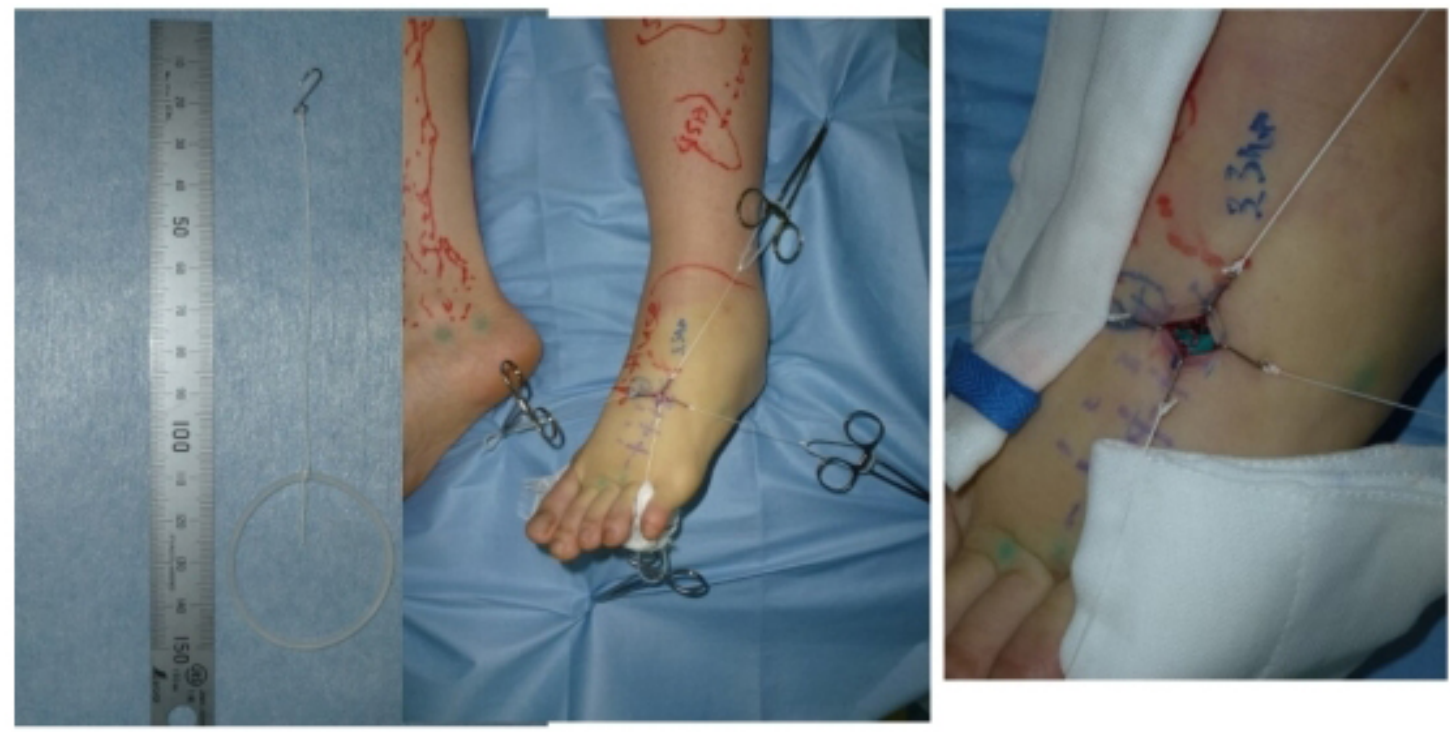

Figure 4. Fixation of the LVA incision using four hook-type retractors. Fixation of the surgical site of LVA. The needle hook-type retractors are useful in LVA to fix the surgical site for suturing small vessels. Four needle hook retractors can keep the stable surgical site for fine procedures of dissection and anastomosis under the operating microscope. Because the incision site of LVA is small (usually 1.0-2.5 cm), larger retractors tend to interfere with the fine procedure. LVA: Lymphaticovenular anastomosis.

\section{Techniques in LVA STEP 2: detection and dissection of small vessels}

The lymphatic vessel adopted in LVA is small (mainly 0.20-0.70 $\mathrm{mm}$ in diameter), which is difficult to detect and to dissect from fibrotic tissues in lymphedema patients even under high magnification of an operating microscope.

Under the tight fixation of the incision using four retractors, Adson forceps with teeth and a microdissector are used to dissect the subcutaneous vein and the lymphatic vessels. Because the lymphatic vessels are transparent and small in diameter, it is easy to lose the detected lymphatic vessels again in fatty tissue. A 3-0 nylon monofilament is used to catch and keep the lymphatic vessel and the smaller subcutaneous vein in dissection without injuring the vessels. In detection and dissection procedure, No. 5 forceps, microscissors, and No. 15 surgical knife are utilized only when the vessels are buried in fibrotic tissues and need to be dissected sharply.

In early lymphedema patients who reveal less sclerosis of lymphatic vessels, the lymphatic vessels are truly transparent and not to be dilatated: detection and anastomosis are sometimes difficult, but dissection is easy. In moderate lymphedema patients who reveal mild to moderate sclerosis of lymphatic vessels, the lymphatic vessels are not actually transparent and to be dilatated: detection, dissection, and anastomosis become relatively easy. In severe lymphedema patients who reveal severe sclerosis of lymphatic vessels, the lymphatic vessels become fragile and easily rupture even under fine procedures of microsurgical dissection. Because of fibrotic changes, dermis and subcutaneous tissues become very hard. In this situation, the microdissector is used as if the surgeon were stabbing the hard soft tissues to reach slightly softer tissues in deep area. Sclerosed lymphatic vessels exist within these fibers, which separate the hard small fatty structures. Because damaged lymphatic vessels also seem to be just fibers in the fibrotic soft tissues, and because degenerated lymphatic vessels are sometimes entwined with fibrous tissue itself, they are easily cut and ruptured by the dissection procedure. 
In these situations, microsurgeons tend to misunderstand that there are no lymphatic vessels at the incision point. However, the truth is that fragile lymphatic vessels are at the site of incision and all lymphatic vessels are ruptured within fibrotic tissues. Even in this situation, careful detection of the ruptured stump of the lymphatic vessels could result in successful LVA by refreshing the stump of the lymphatic vessels after redissection. The subcutaneous veins are also difficult to dissect in these fibrotic tissues. Microsurgeons should not regret finding lymphatic vessels from fibrotic tissues in severe lymphedema patients.

\section{Techniques in LVA STEP 3: anastomosis}

In anastomosing the lymphatic vessel and the subcutaneous vein, the one-hand suture technique is applied. Before the suture, one side tip of the left-hand forceps is used to dilatate the vessel's lumen. If all the vessels are less than $0.20 \mathrm{~mm}$ in diameter with severe sclerosis, or if the lymphatic vessel's intima seems to be easily peeled off by sclerosis, the tip of the right-hand needle (11-0, 12-0, or 12-0s) is utilized as a dilator, which is inserted into the intima.

In progressive lymphedema, creating intima-to-intima anastomosis between the sclerosed lymphatic vessel and the subcutaneous vein is challenging because sclerotic changes of lymphatic vessel make the lumen narrow and fragile. Inaccuracy of the anastomosis in LVA sometimes results in early- or mid-postoperative occlusion. It should be noted that inaccurate procedures in LVA could result in not only early- but also mid-postoperative occlusion because venous thrombosis of LVA is considered to occur less in early phase and more in later phase compared to arterial or venous anastomosis.

In addition, to keep long-term steady flow at LVA, sequential structural disturbance between the lymphatic vessel and the subcutaneous vein should be avoided. At the time of LVA creation, lymphatic vessels are dilatated because of lymphatic fluid excess in the affected limb. However, lymphatic dynamics can change dynamically after LVA: lymphatic flow in LVA is decreasing according to lymphedema improvement in med- postoperative course. The structural disturbance becomes remarkable between lymphatic vessels and subcutaneous veins because lymphatic vessels become smaller over time when the subcutaneous veins do not change in size. Therefore, the relatively smaller subcutaneous vein should be selected for LVA when the lymphatic vessels are abnormally dilatated.

To eliminate early- and mid-postoperative occlusions, improving accuracy of intima-to-intima anastomosing of LVA is important. We previously reported several technical tips in supermicrosurgery, including spear technique to handle small needle in any direction ${ }^{[23]}$ and advanced techniques using onehand suture technique, which facilitate LVA.

\section{Spear technique}

The spear technique is a useful supermicrosurgical suture technique to anastomose small vessels, in which the 11-0, 12-0, or 12-0s needle can be utilized freely from any direction. Proper combination of the angle and the holding part of the needle make microsurgeons' hand sensation possible to feel the lumen of the small vessels and/or lymphatic flow in spear type needle holding. Performance of the LVA with spear technique is described in Supplementary Video 1-3.

\section{Supermicrosurgical LVA style 1: step-by-step suture technique [Supplementary Video 1]}

The first suture is performed using the one-hand suture technique. In this LVA style, the following suture is just put next to the last one. With this technique, the microsurgeon can make each suture by checking the lumen of the vessels until the second last suture. In addition, the microsurgeon can check the lumen of the vessels by inserting the one side tip of the forceps if needed. LVA is completed without concerns of picking 
up the back wall. This technique is beneficial when the lymphatic vessels are not sclerosed severely.

\section{Supermicrosurgical LVA style 2: expanding technique [Supplementary Video 2]}

The first suture is performed using the one-hand suture technique. In this LVA style, the second suture is placed at the opposite side of the first suture. With this technique, the one side tip of the forceps is difficult to insert into the vessels to check the lumen. However, this suture style makes it possible for microsurgeons to feel the lumen more precisely using the one-side suture technique even when the lymphatic vessels are severely sclerosed. After the second suture, even the weak lymphatic flow of sclerosed lymphatic vessels dilatate the anastomosing vessels. In severe lymphedema patients, lymphatic fluid with high viscosity rarely leaks out from the small vessels' gap even when under only two sutures have been built. In this situation, microsurgeons can feel the expanded lumen of the vessel to make additional sutures. Two to three additional sutures are easily added between both arc sides of the first two stitches.

\section{Techniques in LVA STEP 4: closing the incision with wound bed preparation for LVA [Supplementary Video 3]}

Before closing the wound, it is considered that making a comfortable wound bed for LVA is important, not only to keep longtime patency of the anastomosis but also to increase lymphatic flow at the anastomosis. After the fixations of the incision are all released, both sides of the incision are carefully pulled upward. If fibers in the subcutaneous tissues and/or the other lymphatic vessels are three-dimensionally disturbing the created LVA, these structures should be resected [Figure 5]. Because the LVA incision is small in size and lymphatic vessels have collaterals in the extremities, it is more beneficial to cut the disturbing small vessels to increase the lymph-to-venous flow at the LVA for lymphedema improvement. These fibers and/or vessels never play an important role in the lymphatic circulation for patients' lymphedema because they already reveal severe lymphedema before cutting these fibers and/or vessels. Supplementary Video 3 depicts the senior author's technical tricks and tips for LVA including how to make LVA using relatively large vessels, how to check the intraoperative patency of LVA, and how to make a comfortable wound bed for LVA.

\section{SUPERMICROSURGICAL LVA IN THE FUTURE}

The clinical advantage of LVA is the immediate and long-term effect for peripheral lymphedema. In addition, the clinical effects of LVA for generated and acquired lymphatic dysplasia in neonates and infants with pleural effusion, chylothorax, or ascites are reported as a novel surgical treatment for life-threating disorders ${ }^{[24-26]}$. LVA has the possibility to become a first-line surgical treatment for lymphatic diseases in the future because LVA is a minimal invasive surgical treatment with few complications. Furthermore, LVA has pathological advantages that can be easily added to future treatment using lymphangiogenesis technology and regenerative medicine as a combination therapy.

The disadvantage of LVA is the technically difficulty of fine procedures, although technological advancements in operating microscopes including robotic microsurgery are considered to make it possible to perform LVA more easily in the future. Even in this situation, microsurgeons' finger sensation to feel the lymphatic fluid and the lumen must be important to create more and more accurate anastomoses.

The authors believe that most microsurgeons can perform LVA with knowledge of Isao Koshima's supermicrosurgery.

\section{CONCLUSIONS}

LVA is highly effective, minimally invasive surgical treatment for lymphedema. Fundamental and essential techniques for supermicrosurgery facilitate LVA by decreasing risk of postoperative LVA occlusion. 

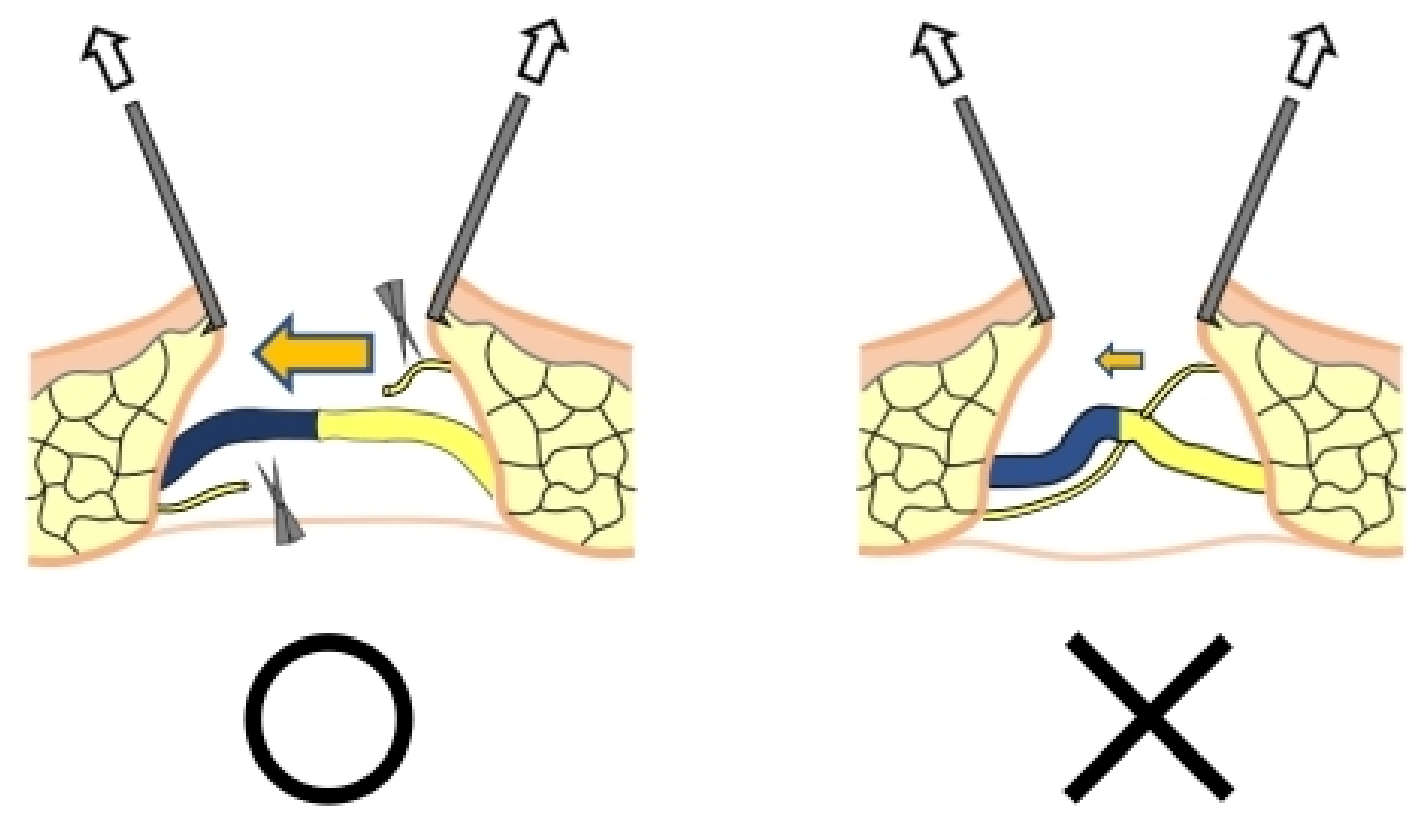

Figure 5. Making a comfortable wound bed for LVA. After the fixations of the incision are all released, both sides of the incision are carefully pulled upward to confirm whether there are any fibers or other lymphatic vessels disturbing the lymph-to-venous flow at the anastomosis. These fibers and/or vessels (right) should be resected to promote lymphatic flow at the LVA to keep the longtime patency with lymphedema improvements.

\section{DECLARATIONS}

\section{Acknowledgments}

The authors thanks Isao Koshima for sharing his knowledge, surgical techniques, passion, and zeal for lymphedema treatment. This article is reported by all our respect to the father of supermicrosurgery, Isao Koshima. And the senior author thanks Yoshiharu, Miyuki, Hanano, Mayoko, and all members of our department for their kind support during preparation of this manuscript. At last, the senior author thanks Takumi Yamamoto for sharing his special technique and knowledge on supermicrosurgical LVA.

\section{Authors' contributions}

Preparation of the article: Seki Y

Teaching importance of developing new ideas and innovations continuously to the senior author:

Kajikawa A

Respect the concept of Isao Koshima's supermicrosugery: Seki Y, Kajikawa A

\section{Availability of data and materials}

Not applicable.

\section{Financial support and sponsorship}

None.

\section{Conflicts of interest}

Both authors declared that there are no conflicts of interest.

\section{Ethical approval and consent to participate}

This study is obtained ethical approval (NO. 4767). 


\section{Consent for publication}

All figures and videos were created by the first author (Seki Y) only for this article. There is no publisher or person who have any copy right on these figures.

\section{Copyright}

(c) The Author(s) 2021.

\section{REFERENCES}

1. Rockson SG, Keeley V, Kilbreath S, Szuba A, Towers A. Cancer-associated secondary lymphoedema. Nat Rev Dis Primers 2019;5:22. DOI PubMed

2. Langer I, Guller U, Berclaz G, et al. Morbidity of sentinel lymph node biopsy (SLN) alone versus SLN and completion axillary lymph node dissection after breast cancer surgery: a prospective Swiss multicenter study on 659 patients. Ann Surg 2007;245:452-61. DOI PubMed PMC

3. Disipio T, Rye S, Newman B, Hayes S. Incidence of unilateral arm lymphoedema after breast cancer: a systematic review and metaanalysis. Lancet Oncol 2013;14:500-15. DOI PubMed

4. Biglia N, Librino A, Ottino MC, Panuccio E, Daniele A, Chahin A. Lower limb lymphedema and neurological complications after lymphadenectomy for gynecological cancer. Int J Gynecol Cancer 2015;25:521-5. DOI PubMed

5. O’Brien BM, Sykes P, Threlfall GN, Browning FS. Microlymphaticovenous anastomoses for obstructive lymphedema. Plast Reconstr Surg 1977;60:197-211. DOI PubMed

6. Koshima I, Inagawa K, Urushibara K, Moriguchi T. Supermicrosurgical lymphaticovenular anastomosis for the treatment of lymphedema in the upper extremities. J Reconstr Microsurg 2000;16:437-42. DOI PubMed

7. Chang DW, Masia J, Garza R 3rd, Skoracki R, Neligan PC. Lymphedema: surgical and medical therapy. Plast Reconstr Surg 2016;138:209S-18S. DOI PubMed

8. Yamamoto T, Yamamoto N, Yoshimatsu H, Narushima M, Koshima I. Factors associated with lymphosclerosis: an analysis on 962 lymphatic vessels. Plast Reconstr Surg 2017;140:734-41. DOI PubMed

9. Yamamoto T, Yamamoto N, Kageyama T, et al. Technical pearls in lymphatic supermicrosurgery. Glob Health Med 2020;2:29-32. DOI PubMed PMC

10. Seki Y, Yamamoto T, Yoshimatsu H, et al. The superior-edge-of-the-knee incision method in lymphaticovenular anastomosis for lower extremity lymphedema. Plast Reconstr Surg 2015;136:665e-75e. DOI PubMed

11. Seki Y, Kajikawa A, Yamamoto T, Takeuchi T, Terashima T, Kurogi N. Single lymphaticovenular anastomosis for early-stage lower extremity lymphedema treated by the superior-edge-of-the-knee incision method. Plast Reconstr Surg Glob Open 2018;6:e1679. DOI PubMed PMC

12. Seki Y, Kajikawa A, Yamamoto T, Takeuchi T, Terashima T, Kurogi N. The dynamic-lymphaticovenular anastomosis method for breast cancer treatment-related lymphedema: creation of functional lymphaticovenular anastomoses with use of preoperative dynamic ultrasonography. J Plast Reconstr Aesthet Surg 2019;72:62-70. DOI PubMed

13. Hayashi A, Hayashi N, Yoshimatsu H, Yamamoto T. Effective and efficient lymphaticovenular anastomosis using preoperative ultrasound detection technique of lymphatic vessels in lower extremity lymphedema. J Surg Oncol 2018;117:290-8. DOI PubMed

14. Seki Y, Yamamoto T, Kajikawa A. Lymphaticovenular anastomosis for breast cancer treatment-related lymphedema: three-line strategy for an optimal outcome. J Plast Reconstr Aesthet Surg 2018;71:e13-4. DOI PubMed

15. Maegawa J, Yabuki Y, Tomoeda H, Hosono M, Yasumura K. Outcomes of lymphaticovenous side-to-end anastomosis in peripheral lymphedema. J Vasc Surg 2012;55:753-60. DOI PubMed

16. Winters H, Tielemans HJP, Verhulst AC, Paulus VAA, Slater NJ, Ulrich DJO. The long-term patency of lymphaticovenular anastomosis in breast cancer-related lymphedema. Ann Plast Surg 2019;82:196-200. DOI PubMed

17. Koshima I, Kawada S, Moriguchi T, Kajiwara Y. Ultrastructural observations of lymphatic vessels in lymphedema in human extremities. Plast Reconstr Surg 1996;97:397-405; discussion 406-7. DOI PubMed

18. Mihara M, Hara H, Hayashi Y, et al. Pathological steps of cancer-related lymphedema: histological changes in the collecting lymphatic vessels after lymphadenectomy. PLoS One 2012;7:e41126. DOI PubMed PMC

19. Masia J, Olivares L, Koshima I, et al. Barcelona consensus on supermicrosurgery. J Reconstr Microsurg 2014;30:53-8. DOI PubMed

20. Hong JP, Pak CJ, Suh HP. Supermicrosurgery in lower extremity reconstruction. Clin Plast Surg 2021;48:299-306. DOI PubMed

21. Yamamoto T, Matsuda N, Doi K, et al. The earliest finding of indocyanine green lymphography in asymptomatic limbs of lower extremity lymphedema patients secondary to cancer treatment: the modified dermal backflow stage and concept of subclinical lymphedema. Plast Reconstr Surg 2011;128:314e-21e. DOI PubMed

22. Yamamoto T, Yamamoto N, Doi K, et al. Indocyanine green-enhanced lymphography for upper extremity lymphedema: a novel severity staging system using dermal backflow patterns. Plast Reconstr Surg 2011;128:941-7. DOI PubMed

23. Seki Y. Lower Extremity Lymphedema. In: Hayash A, Visconti G, editors. Supermicrosurgical lymphaticovenular anastomosis: a practical textbook. Rome: HV Projects; 2020. p. 265-84.

24. Mihara M, Hara H, Shibasaki J, et al. Indocyanine green lymphography and lymphaticovenous anastomosis for generalized lymphatic dysplasia with pleural effusion and ascites in neonates. Ann Vasc Surg 2015;29:1111-22. DOI PubMed

25. Weissler JM, Cho EH, Koltz PF, et al. Lymphovenous anastomosis for the treatment of chylothorax in infants: a novel microsurgical 
approach to a devastating problem. Plast Reconstr Surg 2018;141:1502-7. DOI PubMed

26. Hayashida K, Yamakawa S, Shirakami E. Lymphovenous anastomosis for the treatment of persistent congenital chylothorax in a lowbirth-weight infant: A case report. Medicine (Baltimore) 2019;98:e17575. DOI PubMed PMC 